\title{
THE PSEUDO-CIRCLE IS NOT HOMOGENEOUS
}

\author{
BY \\ JAMES T. ROGERS, JR.
}

In the first two volumes of Fundamenta Mathematica, Knaster and Kuratowski raised the following two questions [15], [16]: (1) If a nondegenerate, bounded plane continuum is homogeneous, is it necessarily a simple closed curve? (2) Does there exist a continuum each subcontinuum of which is indecomposable? Although Knaster settled the second question in 1922 [14], it was to remain until 1948 for the interrelationship of the two questions to become apparent. In that year Moise [18] described an indecomposable, chainable plane continuum which was homeomorphic to each of its proper subcontinua, and Bing [1] showed that this continuum was homogeneous. Bing [2] later showed that this continuum, which Moise called a pseudo-arc, was homeomorphic to Knaster's continuum.

In 1951 Bing [2] showed the existence of uncountably many topologically distinct, hereditarily indecomposable, plane continua. The continuum which he used as a tool in his construction was itself a promising candidate for inclusion in the class of homogeneous, hereditarily indecomposable, plane continua. This circularly chainable continuum had the property that, while it was topologically different from a pseudo-arc, every proper subcontinuum of it was a pseudo-arc; hence it seemed to combine some of the properties of the pseudo-arc and the simple closed curve. Such continua were later referred to as "pseudo-circles" and were discussed by F. B. Jones in [11].

In [20] the author discussed hereditarily indecomposable, circularly chainable continua in general and constructed uncountably many such continua, one of which had the additional property that it could be mapped continuously onto any circularly chainable continuum. The purpose of this paper is to prove that the pseudo-arc is the only homogeneous, hereditarily indecomposable, circularly chainable continuum (Theorem 2). It will first be shown that the pseudo-circle is not homogeneous (Theorem 1) and the methods will be adapted to prove the more general theorem.

C. E. Burgess [11] has asked whether each continuum, each proper subcontinuum of which is homogeneous, is itself homogeneous. Since each proper subcontinuum of a pseudo-circle is a pseudo-arc and hence is homogeneous, this paper answers Burgess' question in the negative.

In this paper a continuum is a nondegenerate, compact, connected subset of a metric space. A map or a mapping is a continuous, single-valued function. A

Received by the editors August 28, 1968.

Copyright (C) 1970, American Mathematical Society 
continuum is indecomposable if it is not the union of two of its proper subcontinua. A continuum is hereditarily indecomposable if each of its subcontinua is indecomposable. A continuum is homogeneous if, for each pair of points in the continuum, there exists a homeomorphism of the continuum onto itself which takes one point into the other. A simple closed curve is the simplest example of a homogeneous plane continuum.

A chain [19] is a finite sequence $L_{1}, L_{2}, \ldots, L_{n}$ of open sets such that $L_{i} \cdot L_{j} \neq \varnothing$ if and only if $|i-j| \leqq 1$. If $L_{1}$ also intersects $L_{n}$, the sequence is called a circular chain. Each $L_{i}$ is called a link. If $\varepsilon$ is a positive number and if the diameter of each link is less than $\varepsilon$, then the sequence is called an $\varepsilon$-chain. A continuum is said to be chainable if, for each positive number $\varepsilon$, the continuum can be covered by an $\varepsilon$-chain. Circularly chainable continua are defined similarly. A pseudo-circle is defined to be a circularly chainable, hereditarily indecomposable, plane continuum which is not chainable. The pseudo-arc [1] is an example of a homogeneous, hereditarily indecomposable, plane continuum which is both circularly chainable and chainable. A solenoid is an example of a homogeneous, indecomposable continuum which is circularly chainable but not chainable.

Independent proofs that the pseudo-circle is not homogeneous were announced simultaneously by the author and L. Fearnley (see Notices Amer. Math. Soc. 15 (1968), p. 942 and p. 943). Fearnley's paper has appeared [7].

1. Degrees of mappings. Let $C$ denote the circle in the plane with center at the origin and circumference two. Orient $C$ so that a definite sense of positive rotation exists. Define the distance between two points of $C$ to be the minimum of the lengths of the two arcs into which these points divide $C$. Let $d$ denote this distance function. Note that $d(C)=1$, where $d(C)$ is defined to be

$$
\sup \{d(x, y) \mid x \in C, y \in C\} \text {. }
$$

Let $A$ be an arc contained in $C$ and let $x$ be an endpoint of $A$. Call $x$ the initial point of $A$ if $x$ is the least point of $A$ with respect to the ordering of $A$ induced by positive rotation. Call $x$ the terminal point of $A$ if $x$ is the greatest point of $A$ with respect to the ordering induced by positive rotation. If $y$ is the initial point of $A$ and $z$ is the terminal point of $A$, then $A$ may also be denoted by [y, $z$ ], a symbol which has the advantage of indicating an ordering as well as a point set. Note that $[y, z]$ and $[z, y]$ are different arcs. The symbol $[y, y]$ is another name for $C$.

Let $f$ be a map of $C$ onto $C$. Associated with the $\operatorname{map} f$ is an integer, called the degree of $f$, which is intuitively the winding number of $f$ or the number of times and the sense of rotation that $f(x)$ rotates around $C$ as $x$ makes one positive rotation. See $[6$, p. 335] for a rigorous definition of degree and for a proof of

LEMMA 1. If $f$ and $g$ are two mappings of $C$ onto $C$, then

$$
\text { degree }(f \circ g)=(\text { degree } f) \cdot(\text { degree } g) \text {. }
$$


Suppose that $f$ and $g$ are two mappings of $C$ onto $C$ and that $\varepsilon$ is a positive number. Define the symbol $f={ }_{\varepsilon} g$ to mean that $d[f(x), g(x)] \leqq \varepsilon$, for each $x$ in $C$.

LEMMA 2. Let $f$ and $g$ be two mappings of $C$ onto $C$, and let $\varepsilon$ be a positive number which is less than one. Then $f={ }_{\varepsilon} g$ implies that the degree of $f$ equals the degree of $g$.

Proof. Since $d(C)=1$ and $\varepsilon<1, f(x)$ and $g(x)$ are not antipodal points, for any $x$ in $C$. Hence [6, p. 316] $f$ and $g$ are homotopic. Then by the theorem of Hopf $[6$, p. 352], the degree of $f$ equals the degree of $g$.

Let $U$ be the class of maps of $C$ onto $C$ which have the property that if $f$ is in $U$ and $x$ is a point of $C$, then $f^{-1}(x)$ has only a finite number of components. Let $p$ be a point in $C$ and let $f$ be an element of $U$. Let $z_{0}, z_{1}, \ldots, z_{k}$ be the boundary points of $f^{-1}(p)$, ordered by positive rotation. Let $z_{k+1}$ be another name for $z_{0}$. Recall that for each $i, 0 \leqq i<k,\left[z_{i}, z_{i+1}\right]$ denotes the arc of $C$ such that $z_{i}$ is the initial point when ordered by positive rotation. If $f \mid\left[z_{i}, z_{i+1}\right]$ is surjective, call $\left[z_{i}, z_{i+1}\right]$ an $A+$ or $A$ - according as the image of $\left[z_{i}, z_{i+1}\right]$ under $f$ emanates from $p$ in the positive or negative direction. If $f \mid\left[z_{i}, z_{i+1}\right]$ is not surjective, call $\left[z_{i}, z_{i+1}\right]$ a $B$. The following lemma, proved in [20], relates these notions to the concept of degree:

LEMma 3. Let $f$ belong to $U$. Then the degree of $f$ equals the number of $A+$ 's of $f$ diminished by the number of $A$-'s of $f$. Hence this number is independent of the point $p$.

Lemma 3 motivates the following definition: If $\left[x_{1}, x_{2}\right]$ is an arc in $C$ such that $f\left(x_{1}\right)=p=f\left(x_{2}\right)$, then the degree of $\left[x_{1}, x_{2}\right]$ with respect to $f$ is the number of $A+$ 's of $f \mid\left[x_{1}, x_{2}\right]$ diminished by the number of $A$-'s of $f \mid\left[x_{1}, x_{2}\right]$. Equivalently, by identifying $x_{1}$ with $x_{2}, f \mid\left[x_{1}, x_{2}\right]$ may be regarded as a map of a circle into a circle. The degree of $f \mid\left[x_{1}, x_{2}\right]$, regarded as a map of a circle into a circle, is the same as the degree of $\left[x_{1}, x_{2}\right]$ with respect to $f$. An obvious, yet important, fact is that Lemma 2 also holds for such restricted mappings with this generalization of degree.

LEMMA 4. Let $f$ and $g$ belong to $U$, and let $\varepsilon$ be a positive number which is less than one. Let $\left[x_{1}, x_{2}\right]$ be an arc in $C$ such that $f\left(x_{1}\right)=f\left(x_{2}\right)=g\left(x_{1}\right)=g\left(x_{2}\right)$. Then $f\left|\left[x_{1}, x_{2}\right]={ }_{\varepsilon} g\right|\left[x_{1}, x_{2}\right]$ implies that the degree of $\left[x_{1}, x_{2}\right]$ with respect to $f$ equals the degree of $\left[x_{1}, x_{2}\right]$ with respect to $g$.

Also following immediately from the definition is the proof of

LEMMA 5. Let $f$ belong to $U$, and let $a, b$, and $c$ be points of $C$ such that $f(a)$ $=f(b)=f(c)$. Then with respect to $f$

$$
\text { degree }[a, b]+\text { degree }[b, c]=\text { degree }[a, c] \text {. }
$$

Let $C_{1}$ and $C_{2}$ be two copies of $C$, and let $f: C_{1} \rightarrow C_{2}$ be an element of $U$. The definition of $A+, A-$, and $B$ partitions the domain of $f$ into a finite collection $W(f, p)$ of $A$ 's and $B$ 's. Let $V=\left\{v_{1}, v_{2}, \ldots, v_{n}\right\}$ be an enumeration of the elements of $W(f, p)$ such that the order of $V$ agrees with positive rotation. For $1 \leqq i \leqq n$, $\left\{v_{1}, v_{2}, \ldots, v_{i}\right\}$ is called an initial segment of $V$, and the arc in $C_{1}$ which is the union 
of $v_{1}, v_{2}, \ldots, v_{i}$ is called an initial segment of $C_{1}$. Similarly $\left\{v_{i}, v_{i+1}, \ldots, v_{n}\right\}$ is called a terminal segment of $V$ and the corresponding arc in $C_{1}$ is called a terminal segment of $C_{1}$. The degree of an initial or terminal segment of $V$ is defined to be the degree of the corresponding arc in $C_{1} . V$ is said to be a canonical ordering of $W(f, p)$ if the degree of any initial segment of $V$ is positive. Note that if $V$ is a canonical ordering and if the degree of $f$ is one, then the degree of any terminal segment is nonpositive. The initial point of $v_{1}$ is said to be an initial point of $C_{1}$ with respect to $f$ and $p$.

LEMMA 6. If $f$ belongs to $U$ and if degree $f=1$, then there exists an unique canonical ordering of $W(f, p)$.

Proof. That there exists a canonical ordering of $W(f, p)$ was proven in [20, Lemma 8]. Suppose there exist two canonical orderings $V=\left\{v_{1}, v_{2}, \ldots, v_{n}\right\}$ and $V^{\prime}=\left\{v_{i}, v_{i+1}, \ldots, v_{n}, v_{1}, \ldots, v_{i-1}\right\}$. Then $\left\{v_{1}, v_{2}, \ldots, v_{i-1}\right\}$, being an initial segment of $V$, has degree greater than or equal to one; in addition, $\left\{v_{i} v_{i+1}, \ldots, v_{n}\right\}$, being an initial segment of $V^{\prime}$, has degree greater than or equal to one. Since the degree of $f$ equals the degree of $\left\{v_{1}, v_{2}, \ldots, v_{i-1}\right\}$ plus the degree of $\left\{v_{i}, v_{i+1}, \ldots, v_{n}\right\}$, the degree of $f \geqq 2$. This contradicts the hypotheses of the lemma.

LEMMA 7. Let $f$ and $g$ be maps with positive degrees which belong to $U$, and let $r$ belong to $C$. Let $q$ be an initial point of a canonical ordering of $W(f, r)$, and let $p$ be an initial point of a canonical ordering of $W(g, q)$. Then $p$ is an initial point of a canonical ordering of $W(f \circ g, r)$.

Proof. Let $z_{0}, z_{1}, \ldots, z_{k}$ be the boundary points of $[f \circ g]^{-1}(r)$, ordered by positive rotation. Let $z_{k+1}$ be another name for $z_{0}$. Since $p$ is one of the $z_{i}$ 's, suppose, without loss of generality, that $p$ is $z_{0}$. The point $p$ is an initial point of an ordering of $W(f \circ g, r)$. If this ordering is not a canonical ordering of $W(f \circ g, r)$, then there exists a smallest positive integer $i$ such that $\left[z_{0}, z_{i}\right]$ has nonpositive degree with respect to $f \circ g$. Let $j$ be the smallest integer greater than or equal to $i$ such that $z$, belongs to $g^{-1}(q)$. Such an integer exists and is not greater than $k+1$. By virtue of the fact that $p$ is an initial point of a canonical ordering of $W(g, q),\left[z_{0}, z_{j}\right]$ has positive degree with respect to $g$. Hence $z_{i}$ and $z_{j}$ are different points. By Lemma 1 , the degree of $\left[z_{0}, z_{j}\right]$ with respect to $f \circ g$ is the product of the degree of $f$ and the degree of $\left[z_{0}, z_{j}\right]$ with respect to $g$. Hence the degree of $\left[z_{i}, z_{j}\right]$ with respect to $f \circ g$ is greater than or equal to the product of the degree of $f$ and the degree of $\left[z_{0}, z_{j}\right]$ with respect to $g$. However, in order for $\left[z_{0}, z_{j}\right]$ to have positive degree with respect to $g,\left[z_{i}, z_{j}\right]$ must be mapped by $g$ onto a terminal segment of the canonical ordering of which $q$ is an initial point. Since any terminal segment of a canonical ordering has degree less than the degree of the map, the degree of $\left[z_{i}, z_{j}\right]$ with respect to $f \circ g$, which is equal to the degree of $\left[g\left(z_{i}\right), g\left(z_{j}\right)\right]$ with respect of $f$, is less than the degree of $f$ multiplied by the degree of $\left[z_{0}, z_{j}\right]$ with respect to $g$. This contradiction shows that the ordering determined by the sequence $z_{0}, z_{1}, \ldots$, $z_{k}$ is a canonical order, and the lemma is proved. 
2. The pseudo-circle as an inverse limit. Let $P$ be a pseudo-circle defined as the common part of a sequence $\left\{D_{i}\right\}$ of circular chains which satisfy the following conditions for each positive integer $i$ :

(1) Each element of $D_{i}$ is the interior of a circle of diameter less than $1 / i$.

(2) The closure of each element of $D_{i+1}$ lies in an element of $D_{i}$.

(3) Each $D_{i}$ covers $P$.

(4) If $E_{i}$ is a proper subchain of $D_{i}$ and if $E_{i+1}$ is a subchain of $D_{i+1}$ which is contained in $E_{i}$, then $E_{i+1}$ is crooked in $E_{i}$.

(5) $D_{1}$ has at least eight links.

(6) Every three consecutive links of $D_{i+1}$ are contained in a link of $D_{i}$, and

(7) $D_{i+1}$ is the union of two chains, $K_{1}$ and $K_{2}$, such that $K_{1}$ runs through $D_{i}$ three times in the positive direction, $K_{2}$ runs through $D_{i}$ two times in the negative direction, the first link of $K_{1}$ is also the last link of $K_{2}$, the first link of $K_{2}$ is also the last link of $K_{1}$, no other link of $K_{1}$ intersects a link of $K_{2}$, and each of the end links of $K_{1}$ is contained in only one (and the same) link of $D_{i}$.

Roughly speaking, (7) says that $D_{i+1}$ runs through $D_{i}$ three times in the positive direction and back two times in the negative direction. Hence $D_{i+1}$ circles one time in $D_{i}$. According to Bing [5, Theorem 4], $P$ is embeddable in the plane. Conditions (1)-(4) are usual conditions in defining a pseudo-circle; conditions (5)-(7) are imposed to simplify the constructions of this paper. Since the pseudocircle is unique [8], we lose no generality in assuming these conditions.

Let $k_{i}$ be the number of links of $D_{i}$. Denote the links of $D_{i}$ by $d_{1}^{i}, d_{2}^{i}, \ldots, d_{k_{i}}^{i}$. Let $X_{i}$ denote $C$ with $k_{i}$ division points, $x_{1}^{i}, x_{2}^{i}, \ldots, x_{k_{i}}^{i}$, dividing $C$ into equal segments. Define a map $\pi_{i}^{i+1}$ taking the division points of $X_{i+1}$ into $X_{i}$ by the following method: Let $\pi_{i}^{i+1}\left(x_{j}^{i+1}\right)$ be $x_{t}^{i}$ if $d_{t}^{i}$ is the only link of $D_{i}$ which contains $d_{j}^{i+1}$, and let $\pi_{i}^{i+1}\left(x_{j}^{i+1}\right)$ be the midpoint of the arc the endpoints of which are $x_{t}^{i}$ and $x_{t+1}^{i}$ if $d_{j}^{i+1}$ is contained in both $d_{t}^{i}$ and $d_{t+1}^{i}$ (there are, of course, two different arcs which have endpoints $x_{t}^{i}$ and $x_{t+1}^{i}$; here we mean the arc which contains no $x_{s}^{i}$ in its interior). Extend $\pi_{i}^{i+1}$ linearly to map all of $X_{i+1}$ onto $X_{i}$. Each $\pi_{i}^{i+1}$ has degree one because of (7).

Define $\pi_{i}^{n}: X_{n} \rightarrow X_{i}$ by composition. Then $\left\{X_{i}, \pi_{i}^{i+1}\right\}$ forms an inverse sequence with limit $X$. Let $u_{j}^{i}=\pi_{i}^{-1}$ (open star of $x_{j}^{i}$ ), and let $U_{i}$ denote the sequence $u_{1}^{i}$, $u_{2}^{i}, \ldots, u_{k_{i}}^{i}$. Each $U_{i}$ is a circular chain covering $X$.

The preceding construction and the following lemma are standard methods of passing from chain constructions to inverse limit representations (see, for example, [10, Lemma 1]).

Lemma 8. $X=\lim \left\{X_{i}, \pi_{i}^{i+1}\right\}$ is homeomorphic to $P$. Furthermore, the circular chains $\left\{U_{i}\right\}$ satisfy conditions (1)-(7) with respect to $X$.

3. Definition of two different points of $X$ and proof that $X$ is not homogeneous. The next lemma, instrumental in defining different points of $X$, relies on (7) in the definition of the pseudo-circle. 
Lemma 9. Let $z$ be a division point of $X_{i}$. Then there exist three division points, $x_{1}, x_{2}, x_{3}$, of $X_{i+1}$ such that $\pi_{i}^{i+1}\left(x_{j}\right)=z, j=1,2,3, x_{1}$ is the initial point of $X_{i+1}$ with respect to $W\left(\pi_{i}^{i+1}, z\right)$, degree $\left[x_{1}, x_{2}\right]=1$, degree $\left[x_{2}, x_{3}\right]=1$, and degree $\left[x_{3}, x_{1}\right]$ $=-1$.

Proof. Consider the rectangle $Q=[0,1] \times[0,3]$ in the plane. Suppose that $g$ is a map of $[0,1]$ onto $[0,3]$ which assumes integral values at zero and one. Define an equivalence relation on $Q$ by $(x, y) \sim\left(x^{\prime}, y^{\prime}\right)$ if and only if $x=x^{\prime}(\bmod 1)$ and $y=y^{\prime}(\bmod 1)$. Then the equivalence relation associates the map $g$ with a map of $C$ onto $C$. Conversely, there exists a map $h:[0,1] \rightarrow[0,3]$ which is associated with $\pi_{i}^{i+1}$ by this equivalence relation.

Let $z_{1} \in[0,1), z_{2} \in[1,2)$, and $z_{3} \in[2,3)$ be the three points of $[0,3)$ which are associated with $z$ under this equivalence relation. Let $x_{1}^{\prime}$ be the largest number in $[0,1]$ such that $h\left(x_{1}^{\prime}\right)=z_{1}$. Let $x_{2}^{\prime}$ be the smallest number in $[0,1]$ such that $x_{2}^{\prime}>x_{1}^{\prime}$ and $h\left(x_{2}^{\prime}\right)=z_{2}$. Let $x_{3}^{\prime}$ be the smallest number such that $h\left(x_{3}^{\prime}\right)=z_{3}$ and $x_{3}^{\prime}>x_{2}^{\prime}$. Numbers $x_{1}^{\prime}, x_{2}^{\prime}$, and $x_{3}^{\prime}$ exist because of condition (7) in the definition of the pseudo-circle.

Let $x_{1}, x_{2}$, and $x_{3}$ be the points of $C$ which correspond to $x_{1}^{\prime}, x_{2}^{\prime}$, and $x_{3}^{\prime}$, respectively, under the equivalence relation. Since $x_{1}^{\prime}$ is the largest number in $[0,1]$ such that $h\left(x_{1}^{\prime}\right)=z_{1}$ and since $h$ is obtained from $\pi_{i}^{i+1}$ which is obtained from a circular chain satisfying condition (7) in the definition of the pseudo-circle, $x_{1}$ is the initial point of $X_{i+1}$ (a sketch of the graph of $h$ helps one to see this point). It follows from the definition of $x_{1}^{\prime}, x_{2}^{\prime}$, and $x_{3}^{\prime}$ that degree $\left[x_{1}, x_{2}\right]=1$ and degree $\left[x_{2}, x_{3}\right]=1$. Since $\pi_{i}^{i+1}$ has degree one, $\left[x_{3}, x_{1}\right]$ must have degree minus one. This proves the lemma.

Two points of the pseudo-circle which are different will now be defined. Let $y_{1}$ be a division point of $X_{1}$. Having chosen $y_{1}, y_{2}, \ldots, y_{k}$, choose $y_{k+1}$ to be the (unique) initial point of $X_{k+1}$ with respect to $\pi_{k}^{k+1}$ and $y_{k}$. The point $y=\left(y_{1}, y_{2}, \ldots\right)$ of $X$ is called a first point of $X$. Let $z_{1}=y_{1}$. Having chosen $z_{1}, z_{2}, \ldots, z_{k}$, choose $z_{k+1}$ as follows: There exist three points $x_{1}, x_{2}$, and $x_{3}$ in $X_{k+1}$ which satisfy the conclusion of Lemma 9 (with $z_{k}=z$ ). Let $z_{k+1}=x_{2}$. The point $z=\left(z_{1}, z_{2}, \ldots\right.$ ) of $X$ is called a second point of $X$.

THEOREM 1. There does not exist a homeomorphism of $X$ onto itself which carries $z$ to $y$; therefore the pseudo-circle is not homogeneous.

Proof. Suppose that there exists a homeomorphism $f$ of $X$ onto itself which carries $z$ to $y$. If $R$ is a circular chain covering $X$, let $f(R)$ denote the circular chain the links of which are images of the links of $R$ under $f$. Choose positive integers $n$ and $m$ such that $f\left(U_{n}\right)$ refines $U_{1}$ and $U_{m}$ refines $f\left(U_{n+1}\right)$. That such integers $m$ and $n$ exist follows from a consideration of Lebesgue numbers and the fact that the homeomorphism $f$ is uniformly continuous. 
Define maps $f_{1}^{\prime}$ taking the division points of $X_{n}$ into $X_{1}$ and $f_{2}$ taking the division points of $X_{m}$ into $X_{n+1}$ by a method similar to that by which the bonding maps $\pi_{n}^{n+1}$ were defined: Let $f_{1}^{\prime}\left(x_{j}^{n}\right)$ be $x_{t}^{1}$ if $u_{t}^{1}$ is the only link of $U_{1}$ which contains $f\left(u_{j}^{n}\right)$, and let $f_{1}^{\prime}\left(x_{j}^{n}\right)$ be the midpoint of the arc of the endpoints of which are $x_{t}^{1}$ and $x_{t+1}^{1}$ if $f\left(u_{j}^{n}\right)$ is contained in both $u_{t}^{1}$ and $u_{t+1}^{1}$ (again we mean the arc which contains no $x_{s}^{1}$ in its interior). Extend $f_{1}^{\prime}$ linearly to map all of $X_{n}$ onto $X_{1}$. Define $f_{2}$ mapping $X_{m}$ onto $X_{n+1}$ similarly.

The maps $f_{1}^{\prime}$ and $f_{2}$ together with the bonding maps $\pi_{1}^{m}$ and $\pi_{n}^{n+1}$ form Diagram 1 . If $\varepsilon$ is a positive number, this diagram is said to be $\varepsilon$-commutative if $f_{1}^{\prime} \pi_{n}^{n+1}$ $f_{2}={ }_{\varepsilon} \pi_{1}^{m}$. We now show that if $\varepsilon$ is the length of a segment of $X_{1}$, then Diagram 1 is $\varepsilon$-commutative.

It is sufficient to prove $\varepsilon$-commutativity for the division points of $X_{m}$ because all maps are extended linearly from the functional values at these points. Let $x_{i}^{m}$ be a division point of $X_{m}$. The link $u_{i}^{m}$ of $U_{m}$ is contained in at most two links of $U_{1}$, say $u_{j}^{1}$ and $u_{j+1}^{1}$. This implies that $\pi_{1}^{m}\left(x_{i}^{m}\right)$ is contained in the arc of $X_{1}$ the endpoints of which are $x_{j}^{1}$ and $x_{j+1}^{1}$.

We must show that $f_{1}^{\prime} \pi_{n}^{n+1} f_{2}\left(x_{i}^{m}\right)$ is contained in the same arc. $U_{m}$ refines $f\left(U_{n+1}\right)$ and hence refines $f\left(U_{n}\right)$. The map $\pi_{n}^{n+1} f_{2}$ carries $x_{i}^{m}$ either into a division point $p$ of $x_{n}$ or into the interior of a segment $\left[p_{1}, p_{2}\right]$ of $X_{n}$, where $p, p_{1}$, and $p_{2}$ correspond to links of $f\left(U_{n}\right)$ containing $u_{i}^{m}$. Since $f\left(U_{n}\right)$ refines $U_{1}$, any link of $f\left(U_{n}\right)$ which contains $u_{i}^{m}$ is contained in $u_{j}^{1}$ and/or $u_{j+1}^{1}$. Hence, in the former case, $f_{1}^{\prime}(p)$ is contained in the arc of $X_{1}$ the endpoints of which are $x_{j}^{1}$ and $x_{j+1}^{1}$. In the latter case $f_{1}^{\prime}\left(p_{1}\right)$ and $f_{1}^{\prime}\left(p_{2}\right)$, and thus $f_{1}^{\prime}\left(\left[p_{1}, p_{2}\right]\right)$, are contained in the arc of $X_{1}$ the endpoints of which are $x_{j}^{1}$ and $x_{j+1}^{1}$. This completes the proof that Diagram 1 is $\varepsilon$-commutative.

Now let $u_{j}^{n}$ denote the link of $U_{n}$ which contains $z_{n}$, and let $u_{t}^{1}$ denote the link of $U_{1}$ which contains $y_{1}$. The link $f\left(u_{j}^{n}\right)$ of $f\left(U_{n}\right)$ is contained in at least one link of $U_{1}$ because $f\left(U_{n}\right)$ refines $U_{1}$; since $f\left(u_{j}^{n}\right)$ contains $f(z)=y$ and since $y$ is contained in only one link of $U_{1}$, the link $f\left(u_{j}^{n}\right)$ is contained only in $u_{t}^{1}$. Hence $f_{1}^{\prime}\left(z_{n}\right)=y_{1}$. Similarly $f_{2}\left(y_{m}\right)=z_{n+1}$. This proves that $\pi_{1}^{m}\left(y_{m}\right)=f_{1}^{\prime} \pi_{n}^{n+1} f_{2}\left(y_{m}\right)=y_{1}$.

Unfortunately, it is not necessarily the case that if $p$ is a division point of $X_{m}$ and if $\pi_{1}^{m}(p)=y_{1}$, then $f_{1}^{\prime} \pi_{n}^{n+1} f_{2}(p)=y_{1}$. A slight modification of $f_{1}^{\prime}$, however, allows us to have this fact. Namely, suppose that $p$ is a point of $X_{m}$ such that $\pi_{1}^{m}(p)=y_{1}$ and $f_{1}^{\prime} \pi_{n}^{n+1} f_{2}(p) \neq y_{1}$. We know that $f_{1}^{\prime} \pi_{n}^{n+1} f_{2}(p)$ differs from $y_{1}$ by less then the length of a segment of $X_{1}$. Let $q=\pi_{n}^{n+1} f_{2}(p)$. We can modify the map $f_{1}^{\prime}$ slightly by "stretching" a small neighborhood $Q$ of $q$ so that the new map takes $q$ to $y_{1}$. This neighborhood should be chosen so that it misses all division points of $X_{n}$ except $q$ and also misses the image under $\pi_{n}^{n+1} f_{2}$ of any other such point $p$ in $X_{m}$. When this is done, note that we have not changed the image of any point in the complement of $Q$ and that the image of any point in $Q$ is moved by less than the length of a segment of $X_{1}$. We modify $f_{1}^{\prime}$ so that if $f_{1}^{\prime} \pi_{n}^{n+1} f_{2}(p)=y_{1}$, then $\pi_{1}^{m}(p)=y_{1}$. If we perform this modification for each such $p$ in $X_{m}$, the new map $f_{1}$ has the property that $\pi_{1}^{m}(p)=y_{1}$ if and only if $f_{1} \pi_{n}^{n+1} f_{2}(p)=y_{1}$. Furthermore 
Lemma 2 implies that the degree of $f_{1}$ is the same as the degree of $f_{1}^{\prime}$. Also Diagram 1 , with $f_{1}^{\prime}$ replaced by $f_{1}$, is $2 \varepsilon$-commutative, where $\varepsilon$ is the length of a segment of $X_{1}$. From now on, when we refer to Diagram 1, we shall always assume that $f_{1}^{\prime}$ has been replaced by $f_{1}$.

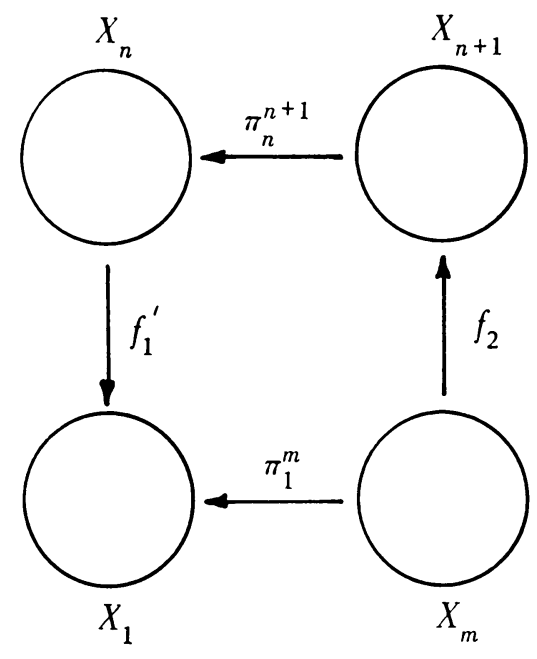

Diagram 1

Since $U_{1}$ has at least eight links and since $X_{1}$ has circumference two, the length of a segment of $X_{1} \leqq 1 / 4$. Since Diagram 1 is $2 \varepsilon$-commutative, where $\varepsilon$ is the length of a segment of $X_{1}$, the maps $\pi_{1}^{m}$ and $f_{1} \pi_{n}^{n+1} f_{2}$ satisfy the hypotheses of Lemma 2. Therefore the degree of $\pi_{1}^{m}$ equals the degree of $f_{1} \pi_{n}^{n+1} f_{2}$. By construction, both $\pi_{1}^{m}$ and $\pi_{n}^{n+1}$ have degree one; accordingly, applying Lemma 1, either degree $f_{1}$ $=$ degree $f_{2}$, or degree $f_{1}=-1=$ degree $f_{2}$.

Let $D_{1}=\left[d_{1}, z_{n+1}\right]$ be the minimal arc in $X_{n+1}$ with $z_{n+1}$ as its terminal point such that the degree of $D_{1}$ with respect to $\pi_{n}^{n+1}$ and $z_{n}$ is nonzero. Let $D_{2}=\left[z_{n+1}, d_{2}\right]$ be the minimal arc in $X_{n+1}$ with $z_{n+1}$ as its initial point such that the degree of $D_{2}$ with respect to $\pi_{n}^{n+1}$ and $z_{n}$ is nonzero. By the manner in which $z_{n+1}$ was chosen, $z_{n+1}$ is the only point common to $D_{2}$ and $D_{1}$. Since both $D_{2}$ and $D_{1}$ are minimal arcs with nonzero degree, each has degree either plus one or minus one.

Let $E=\left[y_{m}, e\right]$ be the minimal arc of $X_{m}$ with $y_{m}$ as its initial point such that either $f_{2}(e)=d_{1}$ or $f_{2}(e)=d_{2}$. Then $f_{1} \pi_{n}^{n+1} f_{2}(e) \subset f_{1} \pi_{n}^{n+1}\left(\left\{d_{1}, d_{2}\right\}\right)=f_{1}\left(z_{n}\right)=y_{1}$. Hence $E$ is an initial segment of $X_{m}$. Thus the degree of $E$ with respect to $\pi_{1}^{m}$ and $y_{1}$ is positive.

It is not possible to pick a point $p$ in $X_{m}$ such that $p$ is not in $E, f_{2}(p)=z_{n+1}$, and degree $\left[y_{m}, p\right]=0$ with respect to $f_{2}$; for this would mean that the degree of [ $\left.y_{m}, p\right]$ with respect to $f_{1} \pi_{n}^{n+1} f_{2}$ is zero. Since $f_{2}(p)=z_{n+1}, f_{1} \pi_{n}^{n+1} f_{2}(p)=y_{1}$. Hence $\pi_{1}^{m}(p)=y_{1}$ because Diagram 1 is commutative over $\left(\pi_{1}^{m}\right)^{-1}\left(y_{1}\right)$. Accordingly, [ $\left.y_{m}, p\right]$ is an initial segment of $X_{m}$ with respect to $\pi_{1}^{m}$ and $y_{1}$, and the degree of 
[ $\left.y_{m}, p\right]$ with respect to $\pi_{1}^{m}$ is positive. This contradicts Lemma 4 and proves the assertion in the first sentence of the paragraph. This assertion will be referred to as Property 1 in the sequel.

The remainder of the proof is divided into two cases. The first case is that degree $f_{1}=1=$ degree $f_{2}$. In this case we show that $f_{2}(e)=d_{2}$.

Suppose $f_{2}(e)=d_{1}$. Let $q_{1}, q_{2}, q_{3}, \ldots, q_{i}, q_{i+1}=y_{m}$ be the division points of the arc $\left[e, y_{m}\right]$ such that $f_{2}\left(q_{j}\right)=z_{n+1}, j=1,2, \ldots, i+1$. Assume the order of the sequence $\left\{q_{i}\right\}$ agrees with positive rotation. We now prove by induction that the degree of [ $y_{m}, q_{j}$ ] with respect to $f_{2}$ is negative $j=1,2, \ldots, i+1$. We show the assertion is true for $j=1$. By Property 1 , the arc $\left[y_{m}, q_{1}\right]$ does not have degree zero. In order that $\left[y_{m}, q_{1}\right]$ have positive degree, it is necessary that the arc $\left[e, q_{1}\right]$ be "wrapped" back around $X_{n+1}$ in the direction of positive rotation. If this happened, some point in the interior of the arc $\left[e, q_{1}\right]$ must be mapped onto $z_{n+1}$ by $f_{2}$. This contradicts the choice of $q_{1}$. Thus the degree of $\left[y_{m}, q_{1}\right]$ with respect to $f_{2}$ is negative.

Assume the assertion is true for $j=1,2, \ldots, k$. Then the degree of $\left[y_{m}, q_{k}\right]$ with respect to $f_{2}$ is negative. The largest value which the degree of $\left[q_{k}, q_{k+1}\right]$ with respect to $f_{2}$ can take is plus one. Thus the degree of $\left[y_{m}, q_{k+1}\right]$ with respect to $f_{2}$ is either negative or zero. The degree of $\left[y_{m}, q_{k+1}\right]$ with respect to $f_{2}$ cannot be zero, by Property 1 . Hence the degree of $\left[y_{m}, q_{k+1}\right]$ with respect to $f_{2}$ is negative. Hence the degree of $f_{2}$, which is equal to the degree of $\left[y_{m}, y_{m}\right]$ with respect to $f_{2}$, is negative. This contradicts the assumptions of Case 1 and completes the proof of the fact that $f_{2}(e)=d_{2}$.

Let $x$ be the initial point of $X_{n+1}$ with respect to $\pi_{n}^{n+1}$ and $z_{n}$. Let $\left[y_{m}, b\right]$ be the minimal arc of $X_{m}$ such that $f_{2}\left(\left[y_{m}, b\right]\right) \supset\left[z_{n+1}, x\right]$. By Property $1, x$ is the only common point of $f_{2}\left(\left[y_{m}, b\right]\right)$ and $\left[x, d_{1}\right]$. Being an initial segment of $X_{m}$ with respect to $\pi_{1}^{m}$ and $y_{1},\left[y_{m}, b\right]$ has positive degree with respect to $\pi_{1}^{m}$. Hence, by Lemma 4, $\left[y_{m}, b\right]$ has positive degree with respect to $f_{1} \pi_{n}^{n+1} f_{2}$. The degree of $\left[y_{m}, b\right]$ with respect to $f_{1} \pi_{n}^{n+1} f_{2}$ equals the degree of $\left[z_{m+1}, x\right]$ with respect to $f_{1} \pi_{n}^{n+1}$. Being a terminal segment of $X_{n+1}$ with respect to $\pi_{n}^{n+1}$ and $z_{n}$, however, implies that $\left[z_{m+1}, x\right]$ has nonpositive degree with respect to $\pi_{n}^{n+1}$ and hence with respect to $f_{1} \pi_{n}^{n+1}$. Therefore the degree of $\left[y_{m}, b\right]$ with respect to $f_{1} \pi_{n}^{n+1} f_{2}$ is nonpositive. This contradiction completes the proof that there do not exist mappings $f_{1}$ and $f_{2}$ with positive degree.

Assume now that the second case holds and that degree $f_{1}=-1=$ degree $f_{2}$. By methods similar to those of the first case, we can show that if $f_{2}(e)=d_{2}$, then $f_{2}$ has nonnegative degree; hence $f_{2}(e)=d_{1}$.

We now show that the degree of $D_{1}$ with respect to $\pi_{n}^{n+1}$ is plus one. Suppose that the degree of $D_{1}$ with respect to $\pi_{n}^{n+1}$ is not plus one. Then, by the definition of $D_{1}$, the degree of $D_{1}$ with respect to $\pi_{n}^{n+1}$ is minus one. Since $f_{1}$ has degree minus one, the degree of $D_{1}$ with respect to $f_{1} \pi_{n}^{n+1}$ is plus one. Since $f_{2}$ maps $E$ onto $D_{1}$ in an order-reversing manner, the degree of $E$ with respect to $f_{1} \pi_{n}^{n+1} f_{2}$ is minus one. $E$ has positive degree with respect to $\pi_{1}^{m}$, however, because $E$ is an 
initial segment of $X_{m}$ with respect to $\pi_{1}^{m}$ and $y_{1}$. This contradiction of Lemma 4 proves that the degree of $D_{1}$ with respect to $\pi_{n}^{n+1}$ is plus one.

Let us review how we picked $z_{n+1}$. By Lemma 6 , there exist three division points $x_{1}, x_{2}, x_{3}$, of $X_{n+1}$ such that $\pi_{n}^{n+1}\left(x_{j}\right)=z_{n}, j=1,2,3, x_{1}$ is the initial point of $X_{n+1}$ with respect to $W\left(\pi_{n}^{n+1}, z_{n}\right)$, degree $\left[x_{1}, x_{2}\right]=1$, degree $\left[x_{2}, x_{3}\right]=1$, and degree $\left[x_{3}, x_{1}\right]=-1$. We chose $z_{n+1}$ to be $x_{2}$. Now let $v=x_{3}$. Then the degree of $\left[z_{n+1}, v\right]$ with respect to $\pi_{n}^{n+1}$ is plus one.

Let $q_{1}=e, q_{2}, q_{3}, \ldots, q_{i+1}=y_{m}$ be the division points of the arc $\left[e, y_{m}\right]$ such that $\pi_{n}^{n+1} f_{2}\left(q_{j}\right)=z_{n}, j=1,2, \ldots, i+1$. Assume the order of the sequence $\left\{q_{i}\right\}$ agrees with positive rotation. We can prove by an argument similar to the one used in Case 1 that the degree of $\left[y_{m}, q_{j}\right]$ with respect to $\pi_{n}^{n+1} f_{2}$ is negative for $j=1,2, \ldots$, $i+1$.

Property 1 guarantees us that for no $q_{j}$ does the arc $\left[y_{m}, q_{j}\right]$ have degree zero with respect to $f_{2}$. Hence let $r$ be the smallest integer such that $f_{2}\left(q_{r}\right)=z_{m+1}$. Let $s$ be the largest integer less than $r$ such that $f_{2}\left(q_{s}\right)=v$. The degree of $\left[y_{m}, q_{r}\right]$ with respect to $\pi_{n}^{n+1} f_{2}$ is minus one. Hence the degree of $\left[y_{m}, q_{r}\right]$ with respect to $f_{1} \pi_{n}^{n+1} f_{2}$ is plus one. Accordingly, the degree of $\left[y_{m}, q_{r}\right]$ with respect to $\pi_{1}^{m}$ is plus one. Therefore the degree of the terminal segment $\left[q_{r}, y_{m}\right]$ with respect to $\pi_{1}^{m}$ is zero. Since $f_{2}$ maps $\left[q_{s}, q_{r}\right]$ onto $\left[v, z_{m+1}\right]$ in an ordering-reversing manner, the degree of $\left[q_{s}, q_{r}\right]$ with respect to $\pi_{n}^{n+1} f_{2}$ equals minus the degree of $\left[z_{m+1}, v\right]$ with respect to $\pi_{n}^{n+1}$ equals minus one. Since the degree of $f_{1}$ is minus one, the degree of $\left[q_{s}, q_{r}\right]$ with respect to $f_{2} \pi_{n}^{n+1} f_{1}$ is plus one. Accordingly, by Lemma 4 , the degree of $\left[q_{s}, q_{r}\right]$ with respect to $\pi_{1}^{m}$ is plus one. Hence with respect to $\pi_{1}^{m}$,

$$
\text { degree } \begin{aligned}
{\left[q_{s}, y_{m}\right] } & =\text { degree }\left[q_{s}, q_{r}\right]+\text { degree }\left[q_{r}, y_{m}\right] \\
& =1+0=1 .
\end{aligned}
$$

Therefore the terminal segment $\left[q_{s}, y_{m}\right]$ has positive degree with respect to $\pi_{1}^{m}$. This contradiction completes the proof of the fact that there do not exist mappings $f_{1}$ and $f_{2}$ which have negative degrees and which complete Diagram 1.

Hence there do not exist mappings $f_{1}$ and $f_{2}$ with either positive or negative degrees. Therefore there is no homeomorphism of $X$ onto itself taking $z$ into $y$; the pseudo-circle, hence, is not homogeneous.

We proceed now to a generalization of Theorem 1. The pseudo-arc is known to be a homogeneous and hereditarily indecomposable plane continuum. That it is circularly chainable can be easily deduced from the fact that it is chainable between each pair of its opposite end points [3]. The substance of the next theorem is that these properties are a characterizaton of the pseudo-arc. Before we prove the theorem, however, a trivial lemma is in order.

LEMMA 10. If $X$ is a circularly chainable continuum, then $X$ is homeomorphic with the inverse limit of an inverse sequence $\left\{X_{n}, \sigma_{n}^{n+1}\right\}$, where each factor space $X_{n}$ is a circumference and one of the following conditions holds for the bonding maps $\sigma_{n}^{n+1}$ : 
(A) The degree of each $\sigma_{n}^{n+1}$ is zero.

(B) The degree of each $\sigma_{n}^{n+1}$ is one.

(C) The degree of each $\sigma_{n}^{n+1}$ is greater than one.

(D) The degree of each $\sigma_{n}^{n+1}$ is minus one.

(E) The degree of each $\sigma_{n}^{n+1}$ is less than minus one.

Proof. One may easily see as a special case of results of J. R. Isbell [10] that a necessary and sufficient condition that $X$ be circularly chainable is that $X$ be homeomorphic to the inverse limit of an inverse sequence $\left\{X_{n}, \sigma_{n}^{n+1}\right\}$, where each factor space $X_{n}$ is a circle and each $\sigma_{n}^{n+1}$ is surjective. The lemma now follows from repeated applications of Lemma 1 together with the following fact about inverse limit sequences: If $\left\{Y_{n}, f_{n}^{n+1}\right\}$ is an inverse sequence with inverse limit $Y$, if $\left\{X_{m(n)}\right\}$ is a subsequence of $\left\{Y_{n}\right\}$, and if $X$ is the inverse limit of the inverse sequence $\left\{X_{n}, f_{m(n)}^{m+1(n)}\right\}$, then $X$ is homeomorphic to $Y$.

THEOREM 2. The pseudo-arc is the only homogeneous, hereditarily indecomposable, circularly chainable continuum.

Proof. Let $X$ be a homogeneous, hereditarily indecomposable, circularly chainable continuum. Then, by Lemma $10, X$ may be represented as the inverse limit of an inverse sequence $\left\{X_{n}, \sigma_{n}^{n+1}\right\}$, where each factor space $X_{n}$ is a circle and the bonding maps $\sigma_{n}^{n+1}$ satisfy one of conditions (A)-(E). Without loss of generality, we shall assume that each bonding map $\sigma_{n}^{n+1}$ has nonnegative degree.

Suppose that each bonding map $\sigma_{n}^{n+1}$ has degree zero. Ingram [9] has shown that if $K$ is a circularly chainable continuum which is defined as the intersection of the circular chains $K_{1}, K_{2}, K_{3}, \ldots$, and if, for each natural number $n$, the circular chain $K_{n+1}$ circles zero times in $K_{n}$ (in the sense of [5]), then $K$ is (linearly) chainable. This condition is equivalent to being the inverse limit of an inverse sequence where the factor spaces are circles and the bonding maps have degree zero. In this case, therefore, $X$ is a homogeneous, chainable continuum. Bing [4] has shown that the only homogeneous, chainable continuum is the pseudo-arc.

Suppose that each bonding map $\sigma_{n}^{n+1}$ has degree one. Bing [5] has shown that if $K$ is a circularly chainable continuum which is defined as the intersection of the circular chains $K_{1}, K_{2}, K_{3}, \ldots$, and if, for each natural number $n$, the circular chain $K_{n+1}$ circles one time in $K_{n}$, then $K$ is embeddable in the plane. Once again this condition is equivalent to being the inverse limit of an inverse sequence where the factor spaces are circles and the bonding maps have degree one. In this case, therefore, $X$ is planar and hence the pseudo-circle; thus, by Theorem $1, X$ is not homogeneous.

Finally, suppose that each bonding map $\sigma_{n}^{n+1}$ has degree greater than one. Bing [5] has shown that such continua are not planar. Hence, in this case, $X$ is one of the continua studied in [20]. Essentially the same argument as that of Theorem 1 may be employed to show that $X$ is not homogeneous. The only difference in the proof is in the fact that an initial point with respect to the bonding map $\sigma_{n}^{n+1}$ is not 
unique; for instance, if the degree of $\sigma_{n}^{n+1}$ were two, then there would exist two points of $X_{n+1}$ which would be initial points of $\sigma_{n}^{n+1}$ with respect to some fixed point in $X_{n}$. The essential fact, however, is that there still exist first points and second points, and it is only necessary to pick a first point and a second point and to apply the proof of Theorem 1 (with appropriate modifications) to show that $X$ is not homogeneous.

Therefore a continuum is a pseudo-arc if and only if it is homogeneous, hereditarily indeomposable, and circularly chainable.

\section{REFERENCES}

1. R. H. Bing, A homogeneous indecomposable plane continuum, Duke Math. J. 15 (1948), 729-742. MR 10, 261.

2. - Concerning hereditarily indecomposable continua, Pacific J. Math. 1 (1951), 43-51. MR 13, 265.

3. —, Snake-like continua, Duke Math. J. 18 (1951), 653-663. MR 13, 265.

4. —_ Each homogeneous nondegenerate chainable continuum is a pseudo-arc, Proc. Amer. Math. Soc. 10 (1959), 345-346. MR 21 \#3818.

5. —, Embedding circle-like continua in the plane, Canad. J. Math. 14 (1962), 113-128. MR 24 \#A1712.

6. J. Dugundji, Topology, Allyn and Bacon, Boston, Mass., 1966. MR 33 \#1824.

7. L. Fearnley, The pseudo-circle is not homogeneous, Bull. Amer. Math. Soc. 75 (1969), 554-558.

8. - The pseudo-circle is unique, Bull. Amer. Math. Soc. 75 (1969), 398-401.

9. W. T. Ingram, Concerning non-planar circle-like continua, Canad. J. Math. 19 (1967), 242-250. MR 35 \#4889.

10. J. R. Isbell, Embeddings of inverse limits, Ann. of Math. (2) 70 (1959), 73-84. MR 21 \#5946.

11. F. B. Jones, On homogeneity, Summary of lectures and seminars, Summer Institute of Set-Theoretic Topology (Madison, Wisconsin, 1955), University of Wisconsin, Madison, 1958, pp. 68-70. MR 21 \#4395.

12. - Certain homogeneous unicoherent indecomposable continua, Proc. Amer. Math. Soc. 2 (1951), 855-859. MR 13, 573.

13. - On a certain type of homogeneous plane continuum, Proc. Amer. Math. Soc. 6 (1955), 735-740. MR 17, 180.

14. B. Knaster, Un continu dont tout sous-contunu est indécomposable, Fund. Math. 3 (1922), 247-286.

15. B. Knaster and C. Kuratowski, Problème 2, Fund. Math. 1 (1920), 223.

16. —, Problème 4, Fund. Math. 2 (1921).

17. S. Mardešić and J. Segal, e-mappings onto polyhedra, Trans. Amer. Math. Soc. 109 (1963), 146-164. MR 28 \#1592.

18. E. E. Moise, An indecomposable plane continuum which is homeomorphic to each of its nondegenerate subcontinua, Trans. Amer. Math. Soc. 63 (1948), 581-594. MR 10, 56.

19. R. L. Moore, Foundations of point set theory, rev. ed., Amer. Math. Soc. Colloq. Publ., vol. 13, Amer. Math. Soc., Providence, R. I., 1962. MR 27 \#709.

20. J. T. Rogers, Jr., Pseudo-circles and universal circularly chainable continua, Illinois J. Math. (to appear).

TULANE UNIVERSITY,

New OrLeans, Louisiana 70118 